\title{
Oceanographic Measurements with Microprocessors
}

\author{
PAUL J. BUCCA AND ROGER W. MEREDITH, MEMBER, IEEE
}

\begin{abstract}
Oceanographic data acquisition is usually done with instrumentation that has taken many hours to configure, program, and learn to use. The advent of self-recording oceanographic data collection systems based on microprocessors has greatly facilitated the acquisition of oceanographic data from ships of opportunity and in harsh environments. Two notable examples are the Ocean Data Equipment (ODEC) model 302A CSTD unit, which measures ocean conductivity, salinity, and temperature as a function of depth from which acoustic sound speed is calculated, and the InterOcean Model S4 current meter, which measures ocean current speed and direction as a function of depth or time. Networked with Apple Corporation's Macintosh microcomputer, the acquisition, processing, and analysis of CSTD and current data become dependable and virtually automatic. Communication between the Macintosh and the oceanographic probes, as well as data processing, is accomplished with commercially available software. Ease of use is accomplished in both instruments through probe features and the Macintosh user interface. Both systems have a proven at-sea track record and are ideally suited to Arctic applications by virtue of their portability, ability to fit through a small 8-in ice hole, and ruggedness. The Macintosh has been modified to run on dc, and the entire suite of instrumentation can be operated entirely from battery power. The commercially available software described here enables the user to collect, reduce, analyze, and even report results in the field minutes after probe recovery.
\end{abstract}

\section{INTRODUCTION}

$\mathrm{N}$ ETWORKING a series of commercially available instrumentation to produce a functional system is often accompanied by many unforeseen problems, which results in a product not ideal for the intended use. The combination of the Ocean Data Equipment (ODEC) model 302A conductivity, salinity, temperature, and depth (CSTD) system [1] and the InterOcean model S4 current meter [2] with Apple Corporation's Macintosh computer [3] is an exception to this rule. This combination provides a user-friendly, rugged suite of instrumentation that can operate entirely on battery power, process data, and produce reports in the field within minutes of probe recovery.

Both the CSTD system and the S4 current meter were chosen for their small physical size, ability to operate from dc, and their reputation as rugged units. The Macintosh was chosen for its ease of use, low cost, small size, and the use of 3.5-in hard-shelled floppy disks, and has been modified to operate from batteries. These attributes are especially valuable

Manuscript received February 9, 1987; revised September 26, 1987. This work was supported by the ASW Environmental Support program, Office of Naval Research Code 132, and the Office of Naval Technology, with technical management provided by NORDA.

The authors are with the Naval Ocean Research and Development Activity, NSTL, MS 39529-5004.

IEEE Log Number 8820770. when data must be collected in such environments as the Arctic, where low weight, dc power usage, and reliability are essential, or onboard a ship of opportunity not designed for oceanographic data collection. Both systems are microprocessor-based programmable self-recording devices, and the problems associated with conventional electromechanical CSTD units (discontinuities in the conductor cable, noisy winch sliprings, etc.) are not present.

Both probes communicate with the Macintosh via an RS232 serial port controlled by software written in Microsoft Corporation's MS-BASIC [4]. The included example terminal emulation program is used for communications with both probes, and an additional subroutine was written to perform the hexadecimal-to-decimal conversions necessary on the data stream output from the S4. Microsoft's integrated application, EXCEL [5], is used for calculating acoustic sound speed, as well as tabulations, statistical analyses, and generating graphic outputs in near real time for on-site analyses and data quality assurance. Only a small amount of programming was necessary to produce a fully operational system.

This paper details the components and interfacing of these microprocessor-based systems and describes field experiences with this instrumentation suite.

\section{CSTD SYSTEM HaRDWARE}

The sound-speed profile in the water column is critical for acoustic propagation modeling and measurement. The CSTD system provides a way to measure temperature, conductivity, and pressure from which salinity and depth are computed. Additionally, sound speed is calculated (to an accuracy of $\pm 0.2 \mathrm{~m} / \mathrm{s}$ ), and all parameters are tabulated and plotted for quality assurance and use in the field. The CSTD profiling system consists of three components, shown in Fig. 1: 1) a probe that contains the underwater sensors, A/D converter electronics, 512-kbyte static RAM buffer for data storage, and an $\mathrm{NiCaD}$ rechargeable battery pack; 2) battery-powered reader/recorder controlled by an 8080 microprocessor that records data from the probe buffer, stores the data on a special audio cassette tape, displays the data on LED's, and outputs the oceanographic data in ASCII format via an RS-232 serial port; and 3) a battery charger to recharge the probe batteries.

The underwater probe has a diameter of 7 in, is 39 in long, weighs $35 \mathrm{lb}$ in air, and has a 1000 -m-depth capability. The small size and low weight are significant factors when operating in the Arctic, from a helicopter, or onboard ships not designed for oceanographic research. The sensor has user-

U.S. Government work not protected by U.S. copyright 


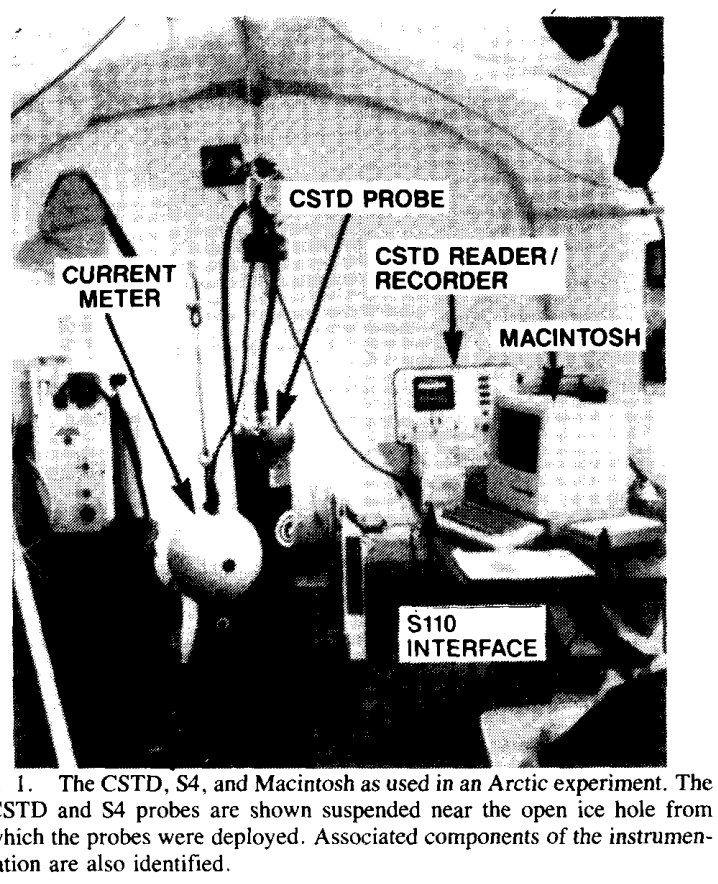

selectable features (such as scanning rate), an upcast disable option, and is self-contained, storing the measurements in RAM. The probe is equipped with a 60 -ms-response platinum thermometer, which allows a relatively fast lowering rate without sensor hysteresis. Data logging is initiated when the conductivity measures greater than $2.4 \mathrm{mS}$ and is terminated when the conductivity falls below $2.4 \mathrm{mS}$ or when the upcast is sensed with the upcast disable circuit enabled. The full sensor specifications are given in [1] and the 512 kbyte of RAM storage in the probe allows a maximum of approximately 10800 scans of conductivity, temperature, and pressure at 5 scans/s.

The battery-powered reader/recorder transfers the data from the sonde RAM to a cassette (containing a special clock track) and displays it on the LED display. The reader/recorder is controlled by an 8080 microprocessor and includes an 8-bit bidirectional data bus. Serial and mode thumb wheels are used to select the various operating modes, including data transfer from the sonde to the reader/recorder, baud-rate selections for the ASCII data transfer from the reader/recorder to the serial port, and data display. The microprocessor in the reader/ recorder calculates salinity and depth and applies previously determined regression equations to the data to compute corrected data in engineering units for display and/or transfer to the serial port.

\section{Current Meter System Hardware}

Horizontal current measurements are becoming increasingly important in determining the deformation of underwater acoustic arrays and in understanding environmental effects on sound propagation in the earth's oceans. The S4 current meter consists of two components, the current meter probe and the S110 electrical interface unit, as shown in Fig. 1. The battery- powered current meter measures current by creating a magnetic field around the spherical probe and sensing the voltage induced by water moving through the magnetic field. This voltage is measured by two pairs of titanium electrodes located symmetrically on each side of the probe. The internal microprocessor determines instrument orientation from a Flux-Gate magnetometer and computes the north and east current vector. The S4 was designed to measure low-speed currents and provides accurate headings at high latitudes. The complete probe specifications are given in [2].

The current vectors can be stored immediately or averaged over preselected times. Data-logging instruments, such as data header entries, sampling frequency, starting time, and averaging time, are downloaded to the $S 4$ via the $\$ 110$ electrical interface unit prior to deployment. The $\mathrm{S} 110$ electrical interface unit communicates via an RS-232 serial port with the Macintosh and is available with a $110 / 120-\mathrm{V}$ ac power supply or with a $12-\mathrm{V} \mathrm{dc}$ power supply. The number of current vector averages can be selected from a maximum of two per second to one per $9 \mathrm{~h}$ and the internal CMOS 64-kbyte static RAM provides enough storage for approximately 20000 current vector samples.

\section{Apple Macintosh}

The Macintosh is an icon-oriented microcomputer that features pull-down command menus and a mouse for controlling software execution. The 3.5-in, hard-shelled floppy disks used by the Macintosh are less fragile than their 5.25-in counterparts and have 800 kbyte of storage. The Macintosh is small, lightweight, transportable, and inexpensive. Its ability to withstand power fluctuations has been proven in use onboard ships and in the Arctic when powered by generators. Recently, the Macintosh was modified to operate from a $12-\mathrm{V}$ battery [6].

Two commercially available software packages are used to store and process the oceanographic data from either of the two probes. Microsoft's MS-BASIC is used to capture data from the Macintosh RS-232 serial port (9600 Bd) and store it on floppy disk, and EXCEL is used to calculate acoustic sound speed, and then to process, tabulate, and plot results. The terminal emulation program, included with MS-BASIC, is used to download and store the CSTD data from the reader/ recorder and upload data-logging instructions to the $\mathrm{S} 4$. The program was modified to calculate depth (meters), current speed (centimeters per second), and direction (degrees from magnetic north) from the S4 hexadecimal output. Several commercially available communications software packages could be used for communicating and processing the data with the Macintosh. MS-BASIC provided a quick and easy means of performing the hexadecimal conversion and byte manipulation necessary for the $S 4$ current meter while maintaining acceptable transfer rates and conversion times. EXCEL is an integrated software package that includes macro commands, a spreadsheet, and a versatile graphics capability. A macro is a program that instructs EXCEL to execute a specified series of tasks or calculations. A macro calculates acoustic sound velocity from salinity, temperature, and depth output by the CSTD probe. Macros also average current data in user-defined 
depth and time increments and tabulate and plot salinity, temperature, and acoustic sound speed as a function of depth, as well as current speed and direction as a function of depth or time. These outputs provide oceanographic data quality assurance and are a powerful analysis tool for supporting acoustical field experiments.

\section{Field EXPERIENCES}

The winch in the background of Fig. 1 is lightweight (100 lb), dc powered, and configured to allow the probes to be deployed vertically tangential to the drum at a variable rate up to $0.3 \mathrm{~m} / \mathrm{s}$. Thus, the entire suite of instrumentation (CSTD probe and reader/recorder, S4 current meter and interface, Macintosh and winch) can be operated in the field entirely from battery power. This instrumentation has been used in the Arctic, and in temperate and subtropical climates, and several techniques have been developed to facilitate operation and data collection. Prior to deployment, it is useful and easy to verify both probes' operability. In the field, it is useful to keep the probe batteries charged between uses and, like any instrumentation that is exposed to saltwater, routine care and maintenance is essential for proper operation. High-quality underwater connectors by Burton (San Diego, California) were special ordered for the CSTD probe to preclude water from shorting out the plug prongs.

Keeping track of where each CSTD data set is located on a single reader/recorder cassette is bothersome. The installation of a tape counter will not alleviate the problem since no LED display is available while the RAM data is transferred to the tape. A hand tabulation of each cast's beginning and ending data points could be maintained to determine data set location on the cassette but would require careful bookkeeping and would be time consuming. This chore is exacerbated by the occasional failure to download data from the probe to the reader/recorder on the first attempt. The best solution is to download each cast on a separate cassette several times.

Use of the S4 probe in the vertical profiling mode is generally limited to deployment from a stationary platform unless the measurement of currents relative to platform movement is relevant (such as deployment from an ice floe to assess the current motion that other sensors experience). When making measurements in the vertical profiling mode, descent rates of approximately $0.2 \mathrm{~m} / \mathrm{s}$ were found to minimize the appearance of artifacts in the data set, and in areas of high current it is necessary to attach deadweight beneath the probe to minimize deflection from the vertical, since the probe is nearly neutrally buoyant in seawater.
One annoyance of the CSTD system is that during a RAM data transfer, a blinking decimal point condition on the LED's alerts the operator either that the RAM data transfer to tape is complete or that a large number of errors have been detected during the transfer. This ambiguity necessitates the manual playback of the data on the LED's to assure successful data transfer. The disadvantage of the $\mathrm{S} 4$ is that the hexadecimal output must be converted to engineering units before the data can be used and thus requires some simple programming, which could just as easily have been done by the $\mathrm{S4}$ microprocessor. Therefore, an external microprocessor must be carried along in the field for downloading data logging instructions and converting the hexadecimal output.

\section{SUMMARY}

Networking the ODEC model 302A CSTD probe, the InterOcean S4 current meter, and the Apple Macintosh computer provides a powerful capability for collecting measurements of ocean salinity and temperature as a function of depth, calculating acoustic sound speed, and measuring current speed and direction as a function of depth or time. This instrumentation suite is ideal for use in the Arctic environment and onboard ships of opportunity not designed for oceanographic research. Unique features include operation on $12 \mathrm{~V}$, compact size, and ease of use. Commercially available software is used to interface the components, to reduce, analyze, plot, and tabulate results in the field for data quality assurance, and to support acoustic propagation experiments. More detailed information on these systems, including program and macro listings, is available from the authors upon request.

\section{REFERENCES}

[1] CSTD Model 302A Technical Manual, Ocean Data Equipment Corp., Middleton, RI, 1986.

[2] S4 Current Meter Manual, InterOcean Systems, Inc., San Diego, CA, 1985.

[3] Macintosh User's Manual, Apple Computer Corp., Cupertino, CA, 1985.

[4] Microsoft BASIC Manual, Microsoft Corp., Bellevue, WA 1985.

[5] EXCEL Manual, Microsoft Corp., Bellevue, WA, 1985.

[6] B. Bricker, private communication, 1987.

\section{t}

Paul J. Bucca, photograph and biography not available at time of publication.

\section{t}

Roger W. Meredith (M'87), photograph and biography not available at time of publication. 